\title{
Effectiveness of BNT162b2 COVID-19 Vaccine in Preventing Severe Symptomatic Infection among Healthcare Workers
}

\author{
Efrén Murillo-Zamora $^{1,2}\left(\mathbb{D}\right.$, Xóchitl Trujillo $^{3}\left(\mathbb{D}\right.$, Miguel Huerta $^{3} \mathbb{D}$, Mónica Riós-Silva $^{3}(\mathbb{D}$ \\ and Oliver Mendoza-Cano ${ }^{4, *(1)}$
}

1 Departamento de Epidemiología, Unidad de Medicina Familiar No. 19, Instituto Mexicano del Seguro Social, Av. Javier Mina 301, Col. Centro, Colima C.P. 28000, Mexico; efren.murilloza@imss.gob.mx

2 Facultad de Medicina, Universidad de Colima, Av. Universidad 333, Col. Las Víboras, Colima C.P. 28040, Mexico

3 Centro Universitario de Investigaciones Biomédicas, Universidad de Colima, Av. 25 de julio 965, Col. Villas San Sebastián, Colima C.P. 28045, Mexico; rosio@ucol.mx (X.T.); huertam@ucol.mx (M.H.); mrios@ucol.mx (M.R.-S.)

4 Facultad de Ingeniería Civil, Universidad de Colima, km. 9 Carretera Colima-Coquimatlán, Coquimatlán, Colima C.P. 28400, Mexico

* Correspondence: oliver@ucol.mx; Tel.: +52-(312)-316-1167

Citation: Murillo-Zamora, E.; Trujillo, X.; Huerta, M.; Riós-Silva, M.; Mendoza-Cano, O. Effectiveness of BNT162b2 COVID-19 Vaccine in Preventing Severe Symptomatic Infection among Healthcare Workers. Medicina 2021, 57, 746. https:// doi.org/10.3390/medicina57080746

Academic Editors: Evangelos I. Kritsotakis and Achilleas Gikas

Received: 9 June 2021

Accepted: 20 July 2021

Published: 23 July 2021

Publisher's Note: MDPI stays neutral with regard to jurisdictional claims in published maps and institutional affiliations.

Copyright: (c) 2021 by the authors. Licensee MDPI, Basel, Switzerland. This article is an open access article distributed under the terms and conditions of the Creative Commons Attribution (CC BY) license (https:// creativecommons.org/licenses/by/ $4.0 /)$.
Abstract: Background and Objectives: This study aims to evaluate the effectiveness of the BNT162b2 COVID-19 (coronavirus disease 2019) in preventing severe symptomatic laboratory-confirmed infection among healthcare workers in a real-world scenario. Materials and Methods: A cross-sectional analysis of a prospective cohort study was conducted. Subjects with onset illness from January to February 2021 were eligible and classified according to the number of vaccine doses received (singleshot, $n=8$; two-shot, $n=12$; unvaccinated, $n=290$ ). Results: The vaccine effectiveness against severe illness was $100 \%$ in the single and two-shot group. The presented results suggest that vaccination reduces the frequency of severe symptomatic COVID-19 in working-age adults. Conclusions: Efforts focusing on maximizing the number of immunized subjects in the study population may reduce associated economic and social burdens.

Keywords: COVID-19 vaccines; BNT162 vaccine; COVID-19; SARS-COV-2

\section{Introduction}

The burden of the coronavirus disease 2019 (COVID-19) caused by the severe acute coronavirus 2 (SARS-CoV-2) in Mexican healthcare workers has been high [1]. Factors determining this scenario include a high prevalence of comorbidities secondary to, among others, unhealthy dietary patterns and sedentary lifestyle in this population segment [2].

Passive immunization plays a critical role in reducing related morbidity and mortality. In Mexico, the BNT162b2 COVID-19 vaccine (Pfizer-BioNTech, New York Cit, NY, USAGermany) was approved by federal authorities on 11 December 2020, and mass scale vaccination among first-line healthcare professionals started by the end of that month [3,4].

However, and to the best of our knowledge, there are no published studies evaluating the vaccination impact on this high-risk group and in the real-world. We aimed to evaluate the effectiveness of BNT162b2 COVID-19 vaccine in preventing severe symptomatic laboratory-confirmed infection in a subset of healthcare workers in a real-world scenario.

\section{Materials and Methods}

We performed a cross-sectional analysis of a prospective cohort study conducted in Mexico and on methods that had been broadly described [5]. Eligible subjects (aged 18 years and above) were laboratory-confirmed COVID-19 cases with illness onset from January to February 2021 and identified as healthcare workers (doctors, nurses, or other healthcare-related positions). 
Laboratory data included a positive reverse-transcription polymerase chain reaction (RT-PCR; Applied Biosystems, Waltham, MA, USA), or rapid antigen test result (Panbio COVID-19 IgG/IgM Rapid Test Device, Abbott Laboratories, Chicago, IL, USA; Standard Q COVID-19 Ag Test, SD Biosensor, Suwon, South Korea) in nasopharyngeal or deep nasal swabs. Severe disease was the main binary outcome, and it was defined by the presence of radiographic or clinical findings of pneumonia requiring hospital admission.

Participants were classified according to COVID-19 vaccination history as the following: unvaccinated (none shot or a single dose received at $<14$ days from acute illness onset), single-shot (one-shot received at 14 or more days from appearance of symptoms) and the two-dose group (adults with complete vaccination scheme). The employed cut-off (14 days) was based on the neutralizing antibodies response after the administration of the mRNA vaccine [6].

Summary statistics were computed, and the significance level was set at $5 \%$. The Local Committee on Health Research (601) of the Mexican Institute of Social Security approved this study (R-2020-601-015).

\section{Results}

Data from 312 individuals were analyzed. The mean age ( \pm standard deviation) of enrolled subjects was $39.3 \pm 11.0$ years old and most of them $(59.3 \%)$ were female. The study profile is summarized in Figure 1.

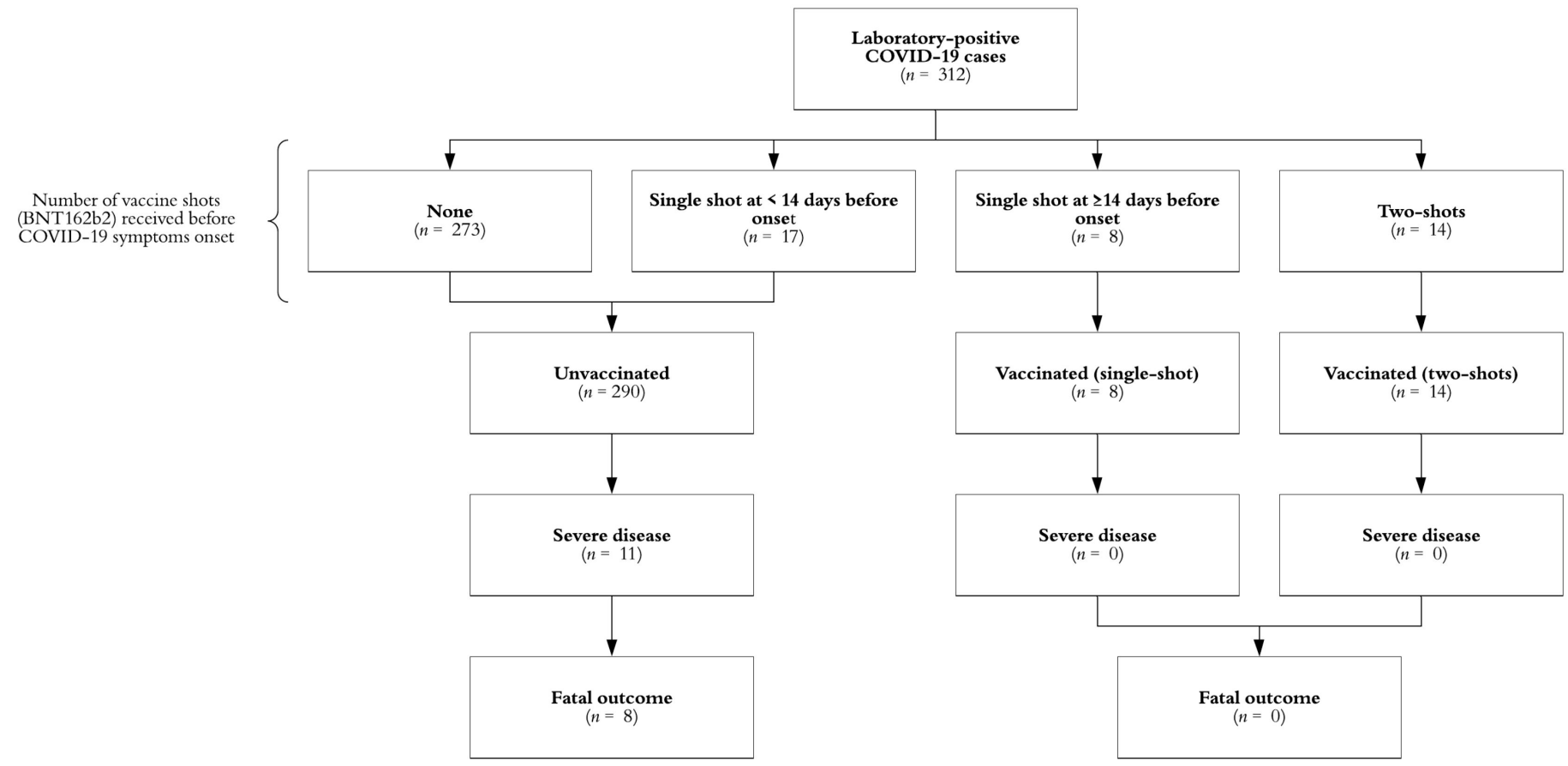

Figure 1. Study profile, Mexico 2021. Abbreviations: COVID-19, coronavirus disease 2019.

Note: Scheme 19. was observed in 3.8\% ( $n=11 / 290)$ of unvaccinated subjects, most of them $(n=10)$ without a single vaccine dose received. The vaccine effectiveness against severe COVID-19 among subjects with a least a single shot was $100 \%$. This scenario was observed, as shown in Table 1, despite that the vaccinated workers were older than the unexposed workers in general $(p=0.007)$. 
Table 1. Characteristics of the study sample for selected variables, Mexico 2021.

\begin{tabular}{|c|c|c|c|c|c|c|c|}
\hline \multirow{3}{*}{ Characteristic } & \multirow{2}{*}{\multicolumn{2}{|c|}{ Unvaccinated }} & \multicolumn{4}{|c|}{ BNT162b2 Vaccination Status } & \multirow{3}{*}{$p$} \\
\hline & & & \multirow{2}{*}{\multicolumn{2}{|c|}{$\begin{array}{c}\text { Single Dose } \\
n=8\end{array}$}} & \multicolumn{2}{|c|}{ Two-Dose } & \\
\hline & \multicolumn{2}{|c|}{$n=290$} & & & \multicolumn{2}{|c|}{$n=14$} & \\
\hline \multicolumn{8}{|l|}{ Gender } \\
\hline Female & 171 & $(59.0)$ & 6 & $(75.0)$ & 8 & $(57.0)$ & 0.651 \\
\hline Male & 119 & $(41.0)$ & 2 & $(25.0)$ & 6 & $(43.0)$ & \\
\hline Age (years) ${ }^{a}$ & \multicolumn{2}{|c|}{$39.0 \pm 10.8$} & \multicolumn{2}{|c|}{$34.1 \pm 10.2$} & \multicolumn{2}{|c|}{$47.5 \pm 12.6$} & 0.007 \\
\hline \multicolumn{8}{|l|}{ Diagnostic method } \\
\hline RT-PCR & 55 & $(19.0)$ & 0 & (0) & 6 & $(42.9)$ & 0.033 \\
\hline Rapid antigenic test & 235 & $(81.0)$ & 8 & $(100)$ & 8 & $(57.1)$ & \\
\hline \multicolumn{8}{|l|}{ COVID-19 severity } \\
\hline Mild-moderate & 279 & $(96.2)$ & 8 & $(100)$ & 14 & $(100)$ & 0.649 \\
\hline Severe & 11 & $(3.8)$ & 0 & $(0)$ & 0 & $(0)$ & \\
\hline \multicolumn{8}{|l|}{ Outcome } \\
\hline Recovery & 282 & $(97.2)$ & 8 & $(100)$ & 14 & $(100)$ & 0.732 \\
\hline Death & 8 & $(2.8)$ & 0 & $(0)$ & 0 & $(0)$ & \\
\hline \multicolumn{8}{|l|}{ Personal history of: } \\
\hline Tobacco use (yes) & 15 & $(5.2)$ & 1 & $(12.5)$ & 0 & (0) & 0.438 \\
\hline Obesity (yes) & 41 & $(14.1)$ & 1 & $(12.5)$ & 2 & $(14.3)$ & 0.991 \\
\hline Type 2 diabetes mellitus (yes) & 17 & $(5.9)$ & 0 & (0) & 2 & (14.3) & 0.707 \\
\hline Arterial hypertension (yes) & 24 & $(8.3)$ & 0 & (0) & 2 & $(14.3)$ & 0.832 \\
\hline Chronic kidney disease (yes) & 1 & $(0.3)$ & 0 & (0) & 0 & $(0)$ & 0.961 \\
\hline
\end{tabular}

Abbreviations: RT-PCR, reverse-transcription polymerase chain reaction; COVID-19, coronavirus disease 2019 Notes: (1) The absolute $(n)$ and relative (\%) frequencies are presented except if the mean is specified; (2) unvaccinated subjects were those with any vaccine shot before onset of COVID-19 symptoms or a single dose at $<14$ days before appearance of symptoms; (3) $p$-value from chi-squared or ANOVA test, as corresponding; (4) severe disease was the main binary outcome, and was defined by the presence of radiographic or clinical findings of pneumonia requiring hospital admission. ${ }^{a}$ Arithmetic mean \pm standard deviation.

No significant differences were observed among the analyzed groups in terms of the frequency of morbidities associated with the risk of a poorer disease outcome.

\section{Discussion}

Our findings suggest that vaccination efforts among Mexican healthcare workers had reduced the burden of COVID-19 in this high-risk group. Frontline healthcare professionals integrate the priority group immunized against the SARS-CoV-2 from December 2020 to February 2021 [7].

In addition to the prevalence of the imported variant 20C/S:452R (B.1.427/9, Epsilon, Irving, TX, USA) in Mexico, recent data revealed the circulation of three variants of interest in Mexico during the pre-vaccination stage. These variants show mutations in the spike protein, P.4 (B.1.1.28.4) and 20B/478K.V1 (B.1.1.222, leading to B.1.1.519), and in the nucleocapsid protein, 20A/N:194L.V2 (B.1.243) [8]. Further research is needed to determine the effectiveness of COVID-19 vaccines against these variants if they are still being transmitted during the post-vaccination stage.

The spread of the Delta variant (B.1.617.2) of SARS-CoV-2, which has already been already in 98 countries, poses a new challenge in the fight against COVID-19 [9]. By mid-July 2021, increasing rates in new COVID-19 cases had been documented in Mexico, coinciding with the relaxation of physical distancing and the spread of the Delta variant.

The limitations of our analysis must be cited. First, the sample size was small, and it was prohibitive to perform a stronger statistical analysis (i.e., evaluate factors associated with illness severity through risk ratios).

Second, we only included symptomatic cases of COVID-19, so the represented spectrum is partial. Third, we lacked data regarding the pre-existence of SARS-CoV-2 immunity, which plays a role in determining the analyzed infection [10]. Despite these limitations, our results are consistent with those previously published in the UK [11] and the USA [12]. 


\section{Conclusions}

These results suggest that vaccination reduces the frequency of severe symptomatic COVID-19 in working-age adults in a real-world scenario. Therefore, efforts focusing on maximizing the number of immunized subjects in the study population may reduce associated economic and social burdens.

Author Contributions: Conceptualization, E.M.-Z. and O.M.-C.; methodology, X.T.; software, M.R.-S.; validation, X.T. and M.H.; formal analysis, M.H.; investigation, M.R.-S.; data curation, O.M.-C.; writing—original draft preparation, E.M.-Z.; writing—review and editing, O.M.-C. and M.H. All authors have read and agreed to the published version of the manuscript.

Funding: This research received no external funding.

Institutional Review Board Statement: The Health Research Committee 601 of the Mexican Institute of Social Security provided approval (R-2020-601-015).

Informed Consent Statement: De-identified data were analyzed and the informed consent to participate was waived.

Data Availability Statement: The datasets generated during and/or analyzed during the current study are available from the corresponding author on reasonable request.

Conflicts of Interest: The authors declare no conflict of interest.

\section{References}

1. Agren, D. Understanding Mexican health worker COVID-19 deaths. Lancet 2020, 396, 807. [CrossRef]

2. Betancourt-Nuñez, A.; Márquez-Sandoval, F.; González-Zapata, L.I.; Babio, N.; Vizmanos, B. Unhealthy dietary patterns among healthcare professionals and students in Mexico. BMC Public Heal. 2018, 18, 1246. [CrossRef] [PubMed]

3. Mexican Institute of Social Security. Press Release 856/2020: With the Application of the Vaccine against COVID-19, the IMSS Takes Part of the Effort of the Health Sector to Overcome the Pandemic. Available online: http://www.imss.gob.mx/prensa/ archivo/202012/856 (accessed on 25 June 2021).

4. Federal Commission for the Protection against Sanitary Risks of Mexico. Authorization for Emergency Use of the CoViD-19 Vaccine. Available online: https:/ / www.gob.mx/cofepris/articulos/la-cofepris-otorga-autorizacion-para-uso-de-emergenciaa-vacuna-para-prevenir-la-enfermedad-por-coronavirus-covid-19 (accessed on 18 April 2021).

5. Murillo-Zamora, E.; Hernandez-Suarez, C.M. Survival in adult inpatients with COVID-19. Public Health 2021, 190, 1-3. [CrossRef] [PubMed]

6. Widge, A.T.; Rouphael, N.G.; Jackson, L.A.; Anderson, E.J.; Roberts, P.C.; Makhene, M.; Chappell, J.D.; Denison, M.R.; Stevens, L.J.; Pruijssers, A.J.; et al. Durability of Responses after SARS-CoV-2 mRNA-1273 Vaccination. N. Engl. J. Med. 2021, 384, 80-82. [CrossRef] [PubMed]

7. Government of Mexico. National Policy for SARS-COV-2 Vaccination to Prevent COVID-19 in Mexico. Available online: https://coronavirus.gob.mx/wp-content/uploads/2021/01/PolVx_COVID_-11Ene2021.pdf (accessed on 16 April 2021).

8. Barona-Gómez, F.; Delaye, L.; Díaz-Valenzuela, E.; Plisson, F.; Cruz-Pérez, A.; Díaz-Sánchez, M.; García-Sepúlveda, C.A.; Sanchez-Flores, A.; Pérez-Abreu, R.; Valencia-Valdespino, F.J.; et al. Phylogenomics and population genomics of SARS-CoV-2 in Mexico during the pre-vaccination stage reveals variants of interest B.1.1.28.4, B.1.1.222 or B.1.1.519 and B.1.243 with mutations in the Spike protein and the Nucleocapsid. medRxiv 2021. [CrossRef]

9. World Health Organization in Mexico. Pandemic Due to Covid-19, Is in a "Very Dangerous" Moment Due to Delta Variant: WHO. Available online: https:/ / coronavirus.onu.org.mx/pandemia-por-covid-19-esta-en-un-momento-muy-peligroso-porvariante-delta-oms (accessed on 10 July 2021).

10. Murillo-Zamora, E.; Mendoza-Cano, O.; Delgado-Enciso, I.; Hernandez-Suarez, C.M. Predictors of severe symptomatic laboratoryconfirmed SARS-CoV-2 reinfection. Public Health 2021, 193, 113-115. [CrossRef] [PubMed]

11. Hall, V.J.; Foulkes, S.; Saei, A.; Andrews, N.; Oguti, B.; Charlett, A.; Wellington, E.; Stowe, J.; Gillson, N.; Atti, A. Effectiveness of BNT162b2 mRNA vaccine against infection and COVID-19 vaccine coverage in healthcare workers in England, multicentre prospective cohort study (the SIREN study). Lancet 2021. [CrossRef]

12. Thompson, M.G.; Burgess, J.L.; Naleway, A.L.; Tyner, H.L.; Yoon, S.K.; Meece, J.; Olsho, L.E.W.; Caban-Martinez, A.J.; Fowlkes, A.; Lutrick, K.; et al. Interim Estimates of Vaccine Effectiveness of BNT162b2 and mRNA-1273 COVID-19 Vaccines in Preventing SARS-CoV-2 Infection Among Health Care Personnel, First Responders, and Other Essential and Frontline Workers-Eight U.S. Locations, December 2020-March 2021. MMWR Morb. Mortal. Wkly. Rep. 2021, 70, 495-500. [PubMed] 